\title{
The optimization of a truss facade
}

\author{
B. Verbeeck ${ }^{1}$, W. P. De Wilde ${ }^{1} \&$ Ph. Samyn ${ }^{2}$ \\ ${ }^{1}$ MeMC, Vrije Universiteit Brussel, Belgium \\ ${ }^{2}$ Samyn and Partners, Belgium
}

\begin{abstract}
This paper presents the study of a facade. One of the aims of the designing team is the minimisation of the weight of the facade. Due to architectural constraints, the geometry of the facade is fixed to a highly statically indeterminate truss. Therefore, the minimisation of the weight of the facade is limited to a section optimisation. The objective function is the indicator of volume. Since the number of members is of the order of 1000, exhaustive search methods are impractical. We used a simple iterative process to find optimal sections. In the first iteration all sections are equal. This allows the forces to flow through the facade as if the facade has a constant stiffness. In the next iterations sections are adapted to the forces that arise from the previous iteration. This method quickly converges to an optimal section layout. The results of this method are corroborated by a genetic algorithm. We find that the truss facade with optimal sections consumes less material than an arch with push rods, that transfer the forces to the arch. Furthermore, the influence of buckling can be ignored.
\end{abstract}

Keywords: morphological indicators, optimization, genetic algorithm, statically indeterminate, truss.

\section{Introduction}

This paper describes a methodology to optimize sections of a (highly) indeterminate truss. It is especially applicable for fixed geometries, where only sections need to be optimized. The method is iterative and consists of updating the stiffness of the members according to forces in that member in the previous iteration. The main advantage of this process is that it consumes considerable less time than an ordinary optimization algorithm (genetic algorithm). Typically it will take the process on the order of 10 iterations before convergence occurs. This means that 


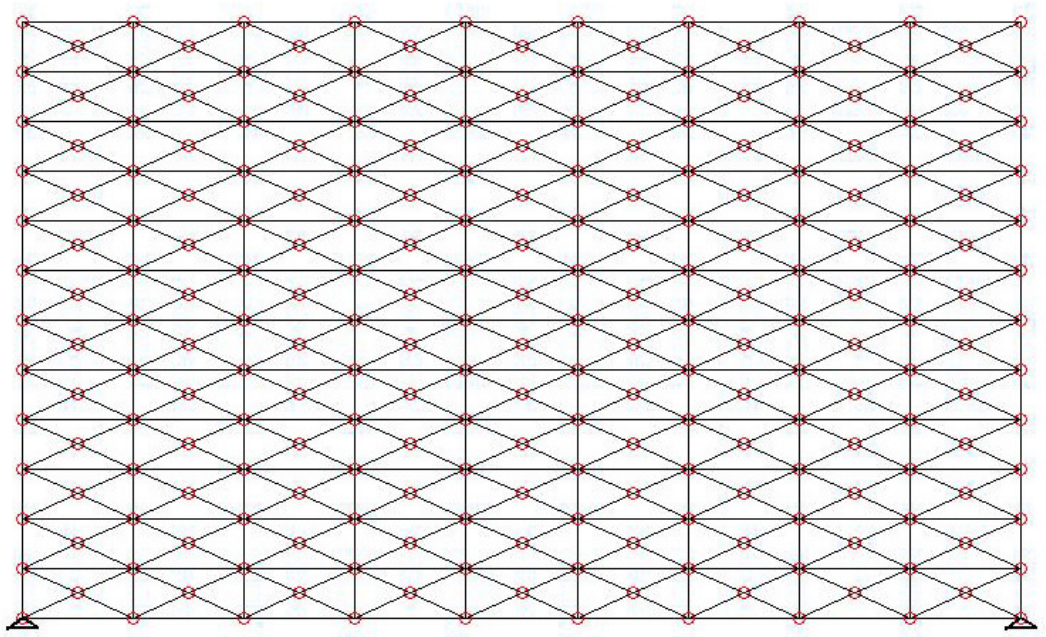

Figure 1: Geometry of the facade, which spans $48.6 \mathrm{~m}$.

only on the order of 10 finite element analyses are performed (compared to $10^{4}$ or more for genetic algorithms). The application of the method is a facade of a large building. A basic geometry is calculated to illustrate the principle. The results for an alternative model is shown and compared to an arch. The "update" method we use was first proposed in [2].

\subsection{Facade}

The facade is composed of 9 by 12 cruciformed modules, which measure respectively $5.4 \mathrm{~m}$ and $3.5 \mathrm{~m}$. Due to the constraint that a train has to pass under the facade, supports are only possible at both extremes (Figure 1). Forces are applied on all nodes. On the top nodes the force is $306 k N$ (roof). The force on all other nodes is $89 k N$.

The other model (Figure 2) has a larger span, but a middle support.

\subsection{Morphological indicators}

In order to compare the different solutions of the facade, an objective function is necessary. The aim of the designers is the minimization of weight (or volume). We therefore use the Morphological Indicators (MI). These dimensionless numbers enable the designer to compare different structural systems on the basis of weight. The most important MI, the Indicator of Volume $W=\frac{\sigma V}{F L}$ allows the comparison of the volume of material for different structural systems. It is the vol- 


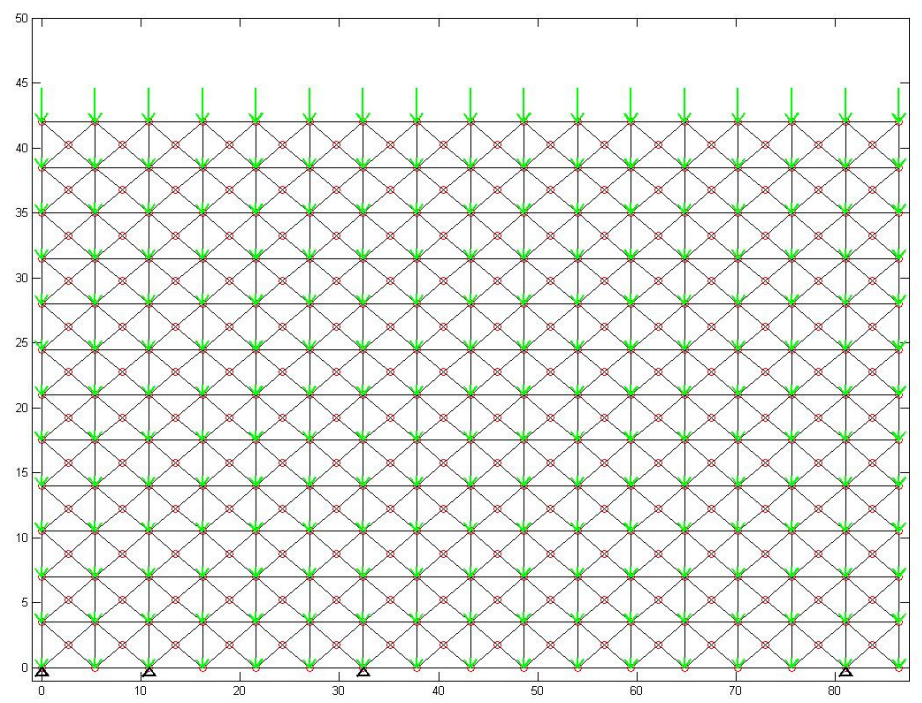

Figure 2: Alternative model (forces are shown).

ume of an isomorphic structure with unit span, with at least one section of each element dimensioned on its unit allowable stress, subjected to a load system with unit resultant. The Displacement Indicator $\Delta=\frac{E \delta}{\sigma L}$ compares the displacement of different structural systems. It is the maximum displacement of an isomorphic structure with unit span in a material with unit Young's Modulus, with at least one section of each element dimensioned on its unit allowable stress, subjected to a load system with unit resultant. The analytical expressions of both $W$ and $\Delta$ have been established in [3] and [2] for trusses, beams, arches, cables, cable stayed structures, masts and frames subjected to a limited number of (simple) load cases and support conditions. For statically determinate structures these Morphological Indicator are only function of the geometrical slenderness $\frac{L}{H}$ if instabilities, self weight and second order effect are neglected. Efficiency curves with respect to minimum volume of used material can be established (Figure 3).

\section{Method}

The first iteration consists of making all sections equal, thus making all modules equally stiff. The system is then solved, using a simple finite element program. The solution can be interpreted as follows: it is a set of sections that is needed to transfer the loads to the supports, if the stiffness is homogeneously distributed over the framework (Figure 4). In reality the stiffness will not be homogeneous, 


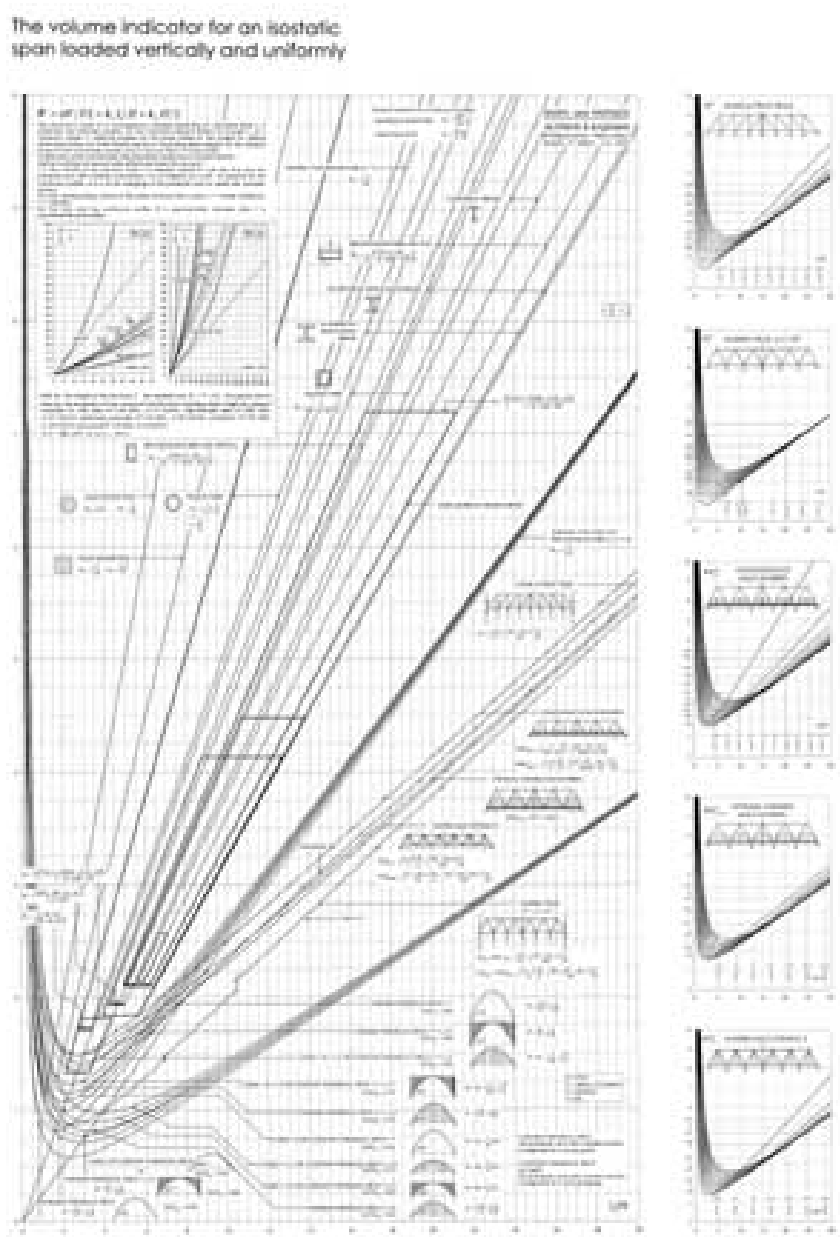

Figure 3: Efficiency curves.

since member sizes vary. Therefore an update of the framework stiffness will yield a more accurate flow of forces. This is done in the second and every successive iteration, until convergence of the objective function $W$.

\section{Results}

The process converges rapidly (Figure 5(a)). We observe that after 1 iteration the value of $W$ is 1.38 . After 10 iterations this value becomes 1.27 . The optimum is reached after 31 iterations $(W=1.26$ ) (Figure 5(b)). The objective function decrease between the first and final (best) iteration is $10 \%$. This decrease becomes 


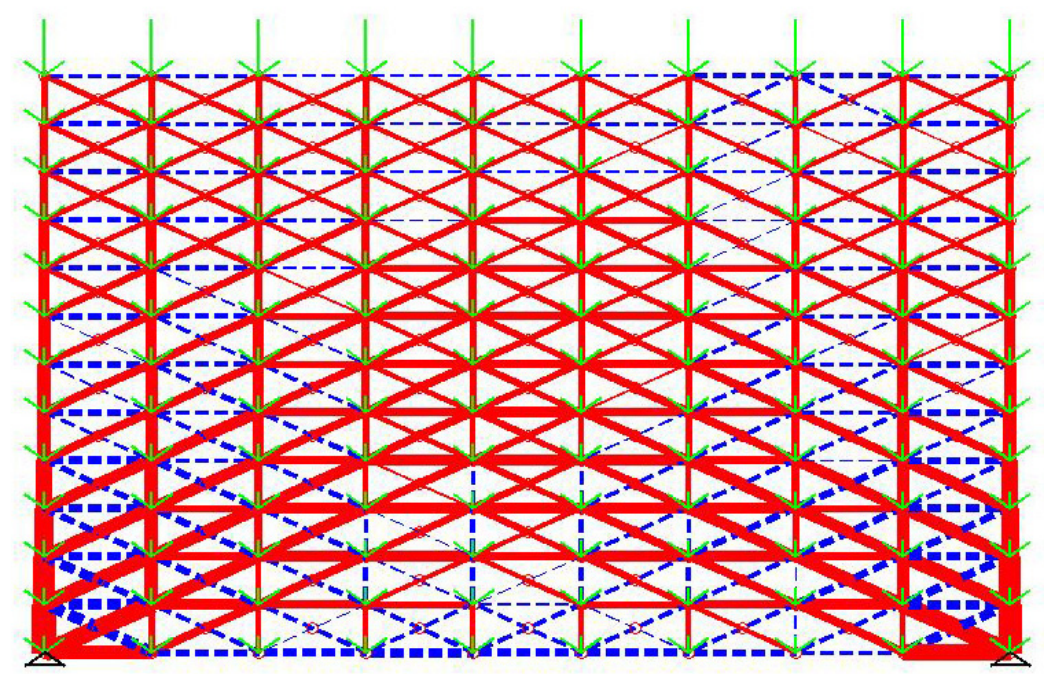

Figure 4: Forces in framework after 1 iteration.

$1 \%$ between iteration 10 and the final one. This means that early on, the process gives a very good estimation of the optimum. Of course this method has to be compared to other optimization techniques. Using the genetic algorithm toolbox of MatLab, a slightly better optimal solution of $W=1.20$ is reached. However, it takes on the order of $10^{4}$ more time to reach this optimum. This has consequences on the design process: because one finite element analysis takes about 2 seconds, the answer of the iterative process is known after 1 minute, whereas the GA yields its result after 5 hours. The advantage of having a slightly better solution does not weigh up against the enormous loss in calculation time, especially during the conceptual design stage.

The results are obtained by incorporating buckling. Optimal solutions vary slightly when buckling is ignored. There are two reasons for this: member lengths are small and sections are buckling efficient, with form factor $\left(\frac{\Omega H^{2}}{I}=8\right.$ with $\Omega$ section, $H$ height and $I$ moment of inertia) low (circular tubes).

The alternative geometry (Figure 6) yields a $W$-value of 1.07.

The previous solution obtained by the "update" iteration is compared to an arch with push rods. According to [3] the value of $W$ of this structural system is 1.13 . This means that the (alternative) truss facade (will transfer the vertical loads more efficiently than an arch with push rods. The inclusion of horizontal (wind) loads will only widen the gap between the two structural systems, because the arch is inefficient at transferring this type of loads. It is not surprising that the truss solutions exhibit an arch shape of its most compressed members and a suspension cable shape of its most tensioned members. 


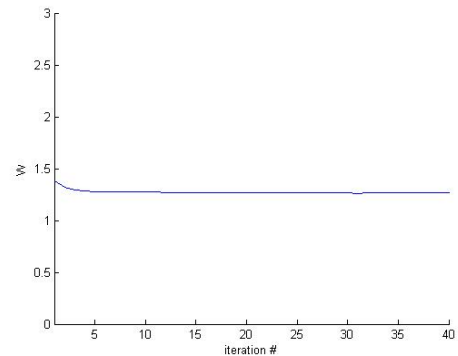

(a) evolution of the objective function

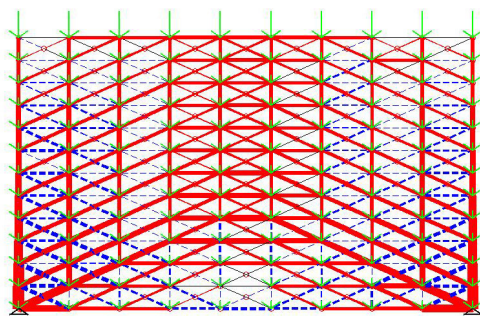

(b) final solution

Figure 5: The result of the iterative process.

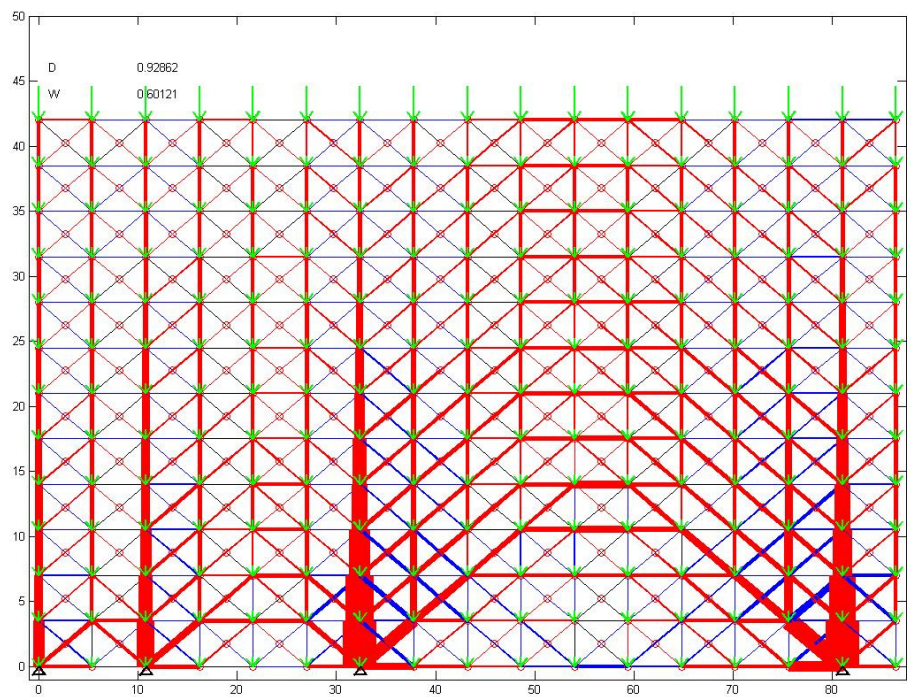

Figure 6: Result for alternative model.

\section{Conclusion and future work}

The method proposed in this paper gives the designer of structures a very fast optimization tool for (highly) statically indeterminate trusses. The process consists of starting with the truss that consists of members with identical sections. In every consecutively step, the sections are adapted to the forces in the members of the 
truss with the previous sections. Within the limits of the conceptual design phase, the results are accurate enough to guide the designer in her/his choices.

With regard to the application, this method yields member sections that make the facade very light. The truss solution is even better than the arch with push rods, wind loads excluded.

A setback of this method is that some boundary conditions (e.g. maximum displacement) are difficult to incorporate. This is due to the method itself. The process can be used for two situations:

1. fixed geometry: only the member sections are to be optimized;

2. truss members used as grid: the truss works as base structure and members that are not useful tend to have sections that converge to zero (Figure 7). This type of optimization echoes the topology optimization method developed by Bendsoe [1]. The problem will be to find the optimal "grid".

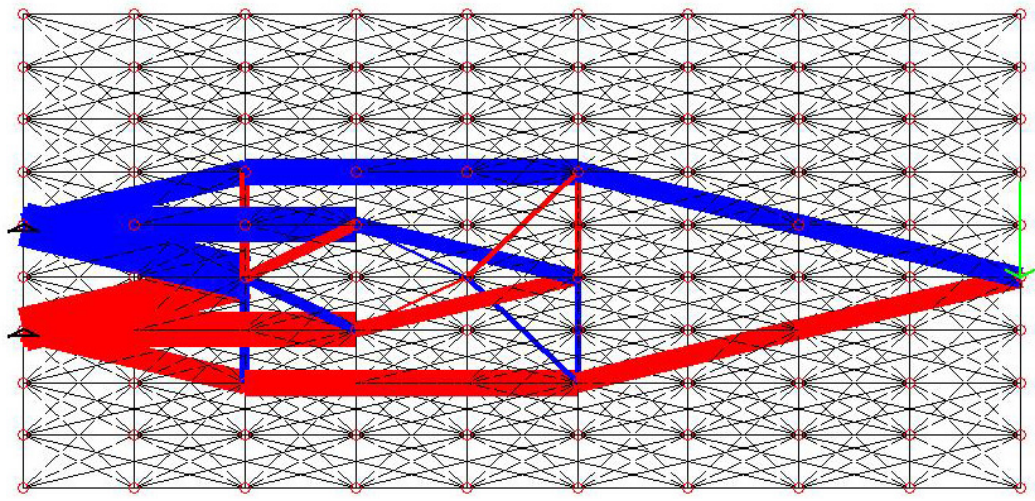

Figure 7: Optimization using "update" method.

\section{References}

[1] M.P. Bendsoe. Optimization of Structural Topology, Shape and Material. Springer, New York, 1995.

[2] P. Latteur. Optimisation et prédimensionnement des treillis, arcs, poutres et câbles sur base d'indicateurs morphologiques. VUB, Brussels, 2004.

[3] Ph. Samyn. Étude de la morphologie des structures à l'aide des indicateurs de volume et de déplacement. Académie Royale de Belgique, Brussels, 2004. 